\title{
Signs activate their written word translation in deaf adults: An ERP study on cross-modal co-activation in German Sign Language
}

Jana Hosemann, Nivedita Mani, Annika Herrmann, Markus Steinbach \& Nicole AltvaterMackensen

\section{Appendix: Complete list of stimulus sentences}

(A) Complete list of stimulus sentences; within-language priming

\begin{tabular}{|c|c|c|}
\hline prime / target & critical and control sentences & phon. \\
\hline $\begin{array}{l}\text { Prim: DOCTOR / MINUTE } \\
\text { Control: POLICE / MINUTE }\end{array}$ & $\begin{array}{l}\text { YESTERDAY ACCIDENT DOCTOR FIVE MINUTE LATE COME } \\
\text { YESTERDAY ACCIDENT POLICE FIVE MINUTE LATE COME }\end{array}$ & loc \\
\hline $\begin{array}{l}\text { Prim: NOON / WATER } \\
\text { Control: EVENING / WATER }\end{array}$ & $\begin{array}{l}\text { DAILY NOON IX }{ }_{1} \text { WATER DRINK SHOULD } \\
\text { DAILY EVENING IX }{ }_{1} \text { WATER DRINK SHOULD }\end{array}$ & hs \\
\hline $\begin{array}{l}\text { Prim: ORANGE / CHAOS } \\
\text { Control: APPLE / CHAOS }\end{array}$ & $\begin{array}{l}\text { SUPERMARKET IX ORANGE SAME CHAOS LOOKS } \\
\text { SUPERMARKET IX APPLE SAME CHAOS LOOKS }\end{array}$ & mov \\
\hline $\begin{array}{l}\text { Prim: ROOM / FAMILY } \\
\text { Control: RESTAURANT / FAMILY }\end{array}$ & $\begin{array}{l}\text { NEXT WEEK ROOM IX FAMILY PARTY ORGANIZE } \\
\text { NEXT WEEK RESTAURANT IX FAMILY PARTY ORGANIZE }\end{array}$ & hs \\
\hline $\begin{array}{l}\text { Pr: ACCEPTANCE / REGISTRATION } \\
\text { Cont: GRAMMAR / REGISTRATION }\end{array}$ & $\begin{array}{l}\text { TALK TOPIC DGS POSS ACCEPTANCE IX REGISTRATION IMPORTANT } \\
\text { TALK TOPIC DGS POSS GRAMMAR IX REGISTRATION IMPORTANT }\end{array}$ & hs \\
\hline $\begin{array}{l}\text { Prim: TREE / TALK } \\
\text { Control: ANIMAL / TALK }\end{array}$ & $\begin{array}{l}\text { TOPIC TREE IX } \text { TALK PREPARE MUST } \\
\text { TOPIC ANIMAL IX } \text { TALK PREPARE MUST }_{1} \text { TALK }\end{array}$ & mov \\
\hline $\begin{array}{l}\text { Prim: STORE / ANIMAL } \\
\text { Control: CHURCH / ANIMAL }\end{array}$ & $\begin{array}{l}\text { USUALLY STORE IX ANIMAL ALLOWED-NEG VISIT } \\
\text { USUALLY CHURCH IX ANIMAL ALLOWED-NEG VISIT }\end{array}$ & mov \\
\hline $\begin{array}{l}\text { Prim: CULTURE / FLOWER } \\
\text { Control: RELIGION / FLOWER }\end{array}$ & $\begin{array}{l}\text { INDIEN POSS CULTURE IX FLOWER IMPORTANT } \\
\text { INDIEN POSS RELIGION IX FLOWER IMPORTANT }\end{array}$ & mov \\
\hline $\begin{array}{l}\text { Prim: MEDICINE / SCIENCE } \\
\text { Control: ART / SCIENCE }\end{array}$ & $\begin{array}{l}\text { CHINA IX MEDICINE SAME SCIENCE OLD } \\
\text { CHINA IX ART SAME SCIENCE OLD }\end{array}$ & loc \\
\hline $\begin{array}{l}\text { Prim: HIKE / PAIN } \\
\text { Control: SPORT / PAIN }\end{array}$ & $\begin{array}{l}\text { YESTERDAY HIKE IX } \text { PAIN SHOULDER } \text { PHTERDAY SPORT IX } 1 \text { PAIN SHOULDER } \\
\text { YESTERD }\end{array}$ & mov \\
\hline $\begin{array}{l}\text { Prim: WORRIES / DREAM } \\
\text { Control: STRESS / DREAM }\end{array}$ & $\begin{array}{l}\text { MANY WORRIES OFTEN DREAM BAD INFLUENCE } \\
\text { MANY STRESS OFTEN DREAM BAD INFLUENCE }\end{array}$ & hs \\
\hline $\begin{array}{l}\text { Prim: BIRTHDAY / ADVANTAGE } \\
\text { Control: WEDDING / ADVANTAGE }\end{array}$ & $\begin{array}{l}\text { SUMMER BIRTHDAY IX ADVANTAGE REASON WEATHER NICE } \\
\text { SUMMER WEDDING IX ADVANTAGE REASON WEATHER NICE }\end{array}$ & mov \\
\hline $\begin{array}{l}\text { Prim: WORK / STONE } \\
\text { Control: HOBBY / STONE }\end{array}$ & $\begin{array}{l}\text { MY GRANDPA POSS WORK IX STONE HAMMER } \\
\text { HL } \\
\text { MY GRANDPA POSS HOBBY IX STONE HAMMER }_{C L}\end{array}$ & mov \\
\hline
\end{tabular}


Prim: INTERVIEW / DIALOGUE Control: MOVIE / DIALOGUE

Prim: SOCIETY / PARTY

Control: COMMUNITY / PARTY

Prim: FUTURE / CRITIQUE Control: 1-YEAR / CRITIQUE

Prim: MATCH / DISCUSSION Con: CHAMPIONSHIP / DISCUSSION

Prim: FUN / MISTAKE

Control: SUCCESS / MISTAKE

Prim: LONDON / BATHROOM

Control: PARIS / BATHROOM

Prim: FEAR / PROBLEM

Control: DEPT / PROBLEM

Prim: STATE / CONFERENCE Cont: UNIVERSITY / CONFERENCE

Prim: TRAINING / EXAMPLE

Control: SCHOOL / EXAMPLE

Prim: GRANDMA / PIG

Control: FATHER / PIG

Prim: HOBBY / ILLNESS

Control: FRUIT / ILLNESS

Prim: INJURY / METAL

Control: INJURY / SAW

Prim: RESTAURANT / DiALOGUE

Control: BAR / DIALOGUE

Prim: PROOF / EXPENSES

Control: RECEIPT / EXPENSES

Prim: PRACTICE / CAR

Control: EXPERIENCE / CAR

Prim: HILL / WOMAN

Control: LAWN / WOMAN

Prim: GRAVE / SHIP

Control: PROBLEM / SHIP

Prim: MATHEMATICS / RAIN

Control: NATURE / RAIN

Prim: WORK / UMBRELLA

Control: SCHOOL / UMBRELLA

Prim: TOWER / UMBRELLA

Control: HOUSE / UMBRELLA
TELEVISION INTERVIEW IX ${ }_{1}$ DIALOGUE DIFFICULT UNDERSTAND TELEVISION MOVIE IX 1 DIALOGUE DIFFICULT UNDERSTAND

hs

DEAF SOCIETY OFTEN PARTY ORGANIZE

DEAF COMMUNITY OFTEN PARTY ORGANIZE

or

$\mathrm{IX}_{1}$ THINK FUTURE IX $\mathrm{X}_{\mathrm{pl}}$ CRITIQUE WITHSTAND MUST

$\mathrm{IX}_{1}$ THINK 1-YEAR IX $\mathrm{pl}_{\mathrm{pl}}$ CRITIQUE WITHSTAND MUST

hs

TOPIC SOCCER MATCH IX DISCUSSION OFTEN LONG

TOPIC SOCCER CHAMPIONSHIP IX DISCUSSION OFTEN LONG

hs

TODAY IX ${ }_{1}$ FUN 10 MISTAKE FIND

TODAY IX 1 SUCCESS 10 MISTAKE FIND

hs

IX 1 VACATION LONDON IX BATHROOM LOOK-FOR

IX 1 VACATION PARIS IX BATHROOM LOOK-FOR

hs

HEIGHT FEAR IX PROBLEM BIG FOR-ME

MANY DEPT IX PROBLEM BIG FOR-ME

hs

THIS YEAR STATE IX CONFERENCE ORGANIZE

THIS YEAR UNIVERSITY IX CONFERENCE ORGANIZE

hs

JOB TRAINING IX EXAMPLE MANY EXIST

JOB SCHOOL IX EXAMPLE MANY EXIST

mov

PAST FARM IX MY GRANDMA OFTEN PIG FEED

PAST FARM IX MY FATHER OFTEN PIG FEED

loc

DIFFERENT HOBBY IX $\mathrm{I}_{\mathrm{pl}}$ ILLNESS PROTECT

DIFFERENT FRUIT IX $\mathrm{pl}_{\mathrm{pl}}$ ILLNESS PROTECT

hs

JOINER POSS INJURY IX METAL CUT

JOINER POSS INJURY IX SAW CUT

mov

WAITER RESTAURANT IX DIALOGUE SIGN CAN

WAITER BAR IX DIALOGUE SIGN CAN

hs

TAX\#RETURN IX ${ }_{1}$ PROOF FOR EXPENSES NEED

TAX\#RETURN IX 1 RECEIPT FOR EXPENSES NEED

hs

DRIVE\#LEARNER MUCH PRACTICE WITH CAR NEED

DRIVE\#LEARNER MUCH EXPERIENCE WITH CAR NEED

mov

YESTERDAY BAVARIA HILL IX WOMAN STAND

YESTERDAY BAVARIA LAWN IX WOMAN STAND

loc

CAPTAIN POSS GRAVE IF SHIP SINK

CAPTAIN POSS PROBLEM IF SHIP SINK

loc

LAW MATHEMATICS SO RAIN ALWAYS FALL-STRAIGTH

LAW NATURE SO RAIN ALWAYS FALL-STRAIGTH CL $_{\text {L }}$

or

YESTERDAY IX ${ }_{1}$ POSS $_{1}$ WORK IX UMBRELLA FORGET

YESTERDAY IX ${ }_{1}$ POSS $_{1}$ SCHOOL IX UMBRELLA FORGET

mov

YESTERDAY IX 1 TOWER IX UMBRELLA FORGET

YESTERDAY IX ${ }_{1}$ HOUSE IX UMBRELLA FORGET 
Prim: DAY / SENTENCE

Control: WEEK / SENTENCE

Prim: DOG / LAUD

Control: CAT / LAUD

Prim: COFFEE / WORK

Control: TOBACCO / WORK

Prim: UMBRELLA / COFFEE

Control: ROOF / COFFEE

Prim: GRIEF / BATH

Control: PROBLEM / BATH

Prim: BANK / STORE

Control: SCHOOL / STORE

Prim: AGENCY / PRINTOUT

Control: OFFICE / PRINTOUT
ABOUT EVERY DAY IX 1 SENTENCE ENGLISH WRITE

ABOUT EVERY WEEK IX ${ }_{1}$ SENTENCE ENGLISH WRITE

mov

MY DOG IX LAUD NEED

MY CAT IX LAUD NEED

loc

BRAZIL POSS COFFEE IX WORK PROCESS LONG

BRAZIL POSS TOBACCO IX WORK PROCESS LONG

mov

IX $_{1}$ TERRACE UMBRELLA IX COFFEE DRINK

IX $_{1}$ TERRACE ROOF IX COFFEE DRINK

mov

LOVE GRIEF IX BATH WARM HELP

LOVE PROBLEM IX BATH WARM HELP

mov

STREET IX BANK NEXT STORE ALREADY CLOSE

STREET IX SCHOOL NEXT STORE ALREADY CLOSE

mov

TOMORROW MAN AGENCY IX PRINTOUT PICK-UP TOMORROW MAN OFFICE IX PRINTOUT PICK-UP

mov

(B) Complete list of stimulus sentences; cross-language priming

prime / target

Prim: Engel / Angel

Control: Mann / Angel

Prim: Raum / Baum

Control: Haus / Baum

Prim: Wein / Bein

Control: Sekt / Bein

Prim: Tier / Bier

Control: Kind / Bier

Prim: Tuch / Buch

Control: Tisch / Buch

Prim: Mutter / Butter

Control: Vater / Butter

Prim: Dach / Schach

Control:Tür / Schach

Prim: Kampf / Dampf

Control: Training / Dampf

Prim: Dieb / Sieb

Control: Mann / Sieb

Prim: Fahne / Sahne

Control: Hemd / Sahne critical and control sentences

MUSEUM IX PICTURE CONTENT TOPIC ANGEL WITH FISHING-ROD HANG MUSEUM IX PICTURE CONTENT TOPIC MAN WITH FISHING-ROD HANG

ARCHITECT WISH ROOM IX TREE PLACE $\mathrm{CL}_{\mathrm{C}}$ ARCHITECT WISH HOUSE IX TREE PLACE $\mathrm{CL}_{\mathrm{C}}$

YESTERDAY RESTAURANT IX WINE IX LEG SPILL $_{\mathrm{CL}}$ YESTERDAY RESTAURANT IX CHAMPAGNE IX ${ }_{1}$ LEG SPILL $_{\mathrm{CL}}$

USUALLY ANIMAL IX BEER DRINK LIKE-NEG

USUALLY CHILD IX BEER DRINK LIKE-NEG

MAGUS PERSON CLOTH IX BOOK LAY-BENEATH ${ }_{\mathrm{CL}}$ MAGUS PERSON TABLE IX BOOK LAY-BENEATH ${ }_{\mathrm{CL}}$

REFRIGERATOR POSS ${ }_{1}$ MOTHER IX BUTTER FETCH REFRIGERATOR POSS ${ }_{1}$ FATHER IX BUTTER FETCH

HOUSE POSS ${ }_{3}$ ROOF LIKE CHESS PATTERN LOOK HOUSE POSS 3 DOOR LIKE CHESS PATTERN LOOK

LAST-TIME BOX FIGHT IX STEAM MUCH

LAST-TIME BOX TRAINING IX STEAM MUCH

YESTERDAY KITCHEN IX OLD THIEF THREE SIEVE STEAL YESTERDAY KITCHEN IX OLD MAN THREE SIEVE STEAL

POSS $_{1}$ SOCCER FLAG IX CREAM SMACK $_{\mathrm{CL}}$ POSS $_{1}$ SOCCER SHIRT IX CREAM SMACK $_{\mathrm{CL}}$ 
Prim: Narbe / Farbe Control: Hose / Farbe

Prim: Tisch / Fisch

Control: Küche / Fisch

Prim: Gabel / Kabel

Control: Messer / Kabel

Prim: Bericht / Gericht

Control: Antrag / Gericht

Prim: Paar / Haar

Control: Geschwister / Haar

Prim: Hahn / Zahn

Control: Vogel / Zahn

Prim: Wand / Hand

Control: Tisch / Hand

Prim: Land / Sand

Control: Ort / Sand

Prim: Mantel / Hantel

Control: Tasche / Hantel

Prim: Hase / Nase

Control: Katze / Nase

Prim: Haus / Maus

Control: Küche / Maus

Prim: Hose / Rose

Control: Hemd / Rose

Prim: Nummer / Kummer

Control: Arzt / Kummer

Prim: Hund / Mund

Control: Katze / Mund

Prim: Mut / Hut

Control: Angst / Hut

Prim: Wette / Kette

Control: Gespräch / Kette

Prim: Kind / Wind

Control: Bruder / Wind

Prim: Koch / Loch

Control: Mann / Loch

Prim: Topf / Kopf

Control: Schüssel / Kopf

Prim: Liege / Ziege

Control: Tisch / Ziege
T-I-M POSS ${ }_{3}$ SCAR IX COLOR SLOWLY CHANGE

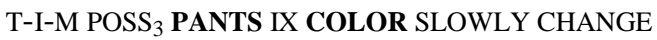

DINNER POSS $_{1}$ MOTHER TABLE IX FISH CUT CL $_{1}$ DINNER POSS $_{1}$ MOTHER KITCHEN IX FISH CUT $\mathrm{CL}_{\mathrm{C}}$

POLICE INFO $_{\text {PL }}$, PLEASE METAL FORK IX WIRE CONTACT MUST-NEG

POLICE INFO $_{\mathrm{PL}}$, PLEASE METAL KNIFE IX WIRE CONTACT MUST-NEG

POSS $_{1}$ INSURANCE ACCIDENT REPORT FOR COURT REFUSE

POSS $_{1}$ INSURANCE ACCIDENT REQUEST FOR COURT REFUSE

BERLIN IX EXIST COUPLE BOTH HAIR RED

BERLIN IX EXIST SIBLINGS BOTH HAIR RED

USUALLY ROOSTER IX $\mathrm{PL}_{\mathrm{PL}}$ TOOTH EXIST-NEG

USUALLY BIRD IX $\mathrm{PL}_{\mathrm{PL}}$ TOOTH EXIST-NEG

KINDERGARTEN IX WALL MANY HAND PRINT ${ }_{C L}$ KINDERGARTEN IX PICTURE MANY HAND PRINT ${ }_{\mathrm{CL}}$

EGYPT IX COUNTRY MUCH SAND EXIST

EGYPT IX AREA MUCH SAND EXIST

SPORT FINISH, MAN POSS 3 COAT IX BARBELL POCKET $\mathrm{CL}$ SPORT FINISH, MAN POSS 3 BAG IX BARBELL POCKET $\mathrm{CL}$

POSS $_{1}$ DAUGHTER POSS $_{3 \mathrm{a}}$ RABBIT IX NOSE SCRATCH $\mathrm{CL}_{\mathrm{CL}}$ POSS $_{1}$ DAUGHTER POSS $_{3 \mathrm{a}}$ CAT IX NOSE SCRATCH $\mathrm{CL}_{\mathrm{L}}$

LAST WEEK POSS ${ }_{1}$ HOUSE IX MOUSE HIDE

LAST WEEK POSS ${ }_{1}$ KITCHEN IX MOUSE HIDE

WEDDING-DAY POSS ${ }_{1}$ PARTNER POSS $_{3 \mathrm{a}}$ PANTS IX $_{1}$ ROSE PUT-IN

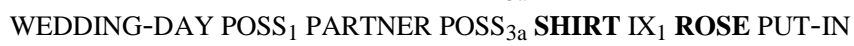

POSS $_{1}$ PARTNER IX $_{3 \mathrm{a}}$ SICK. IX 1 NUMBER FOR GRIEF CALL POSS $_{1}$ PARTNER IX $_{3 \mathrm{a}}$ SICK. IX 1 DOCTOR FOR GRIEF CALL

POSS $_{1}$ GRANDPA POSS 3 DOG IX MOUTH STINK

POSS $_{1}$ GRANDPA POSS 3 CAT IX MOUTH STINK

YESTERDAY IX ${ }_{1}$ VERY COURAGE GO HAT STRANGE BUY

YESTERDAY IX ${ }_{1}$ VERY FEAR GO HAT STRANGE BUY

YESTERDAY WE-DUAL STRANGE BET TOPIC CHAIN IX STABLE OR BREAK YESTERDAY WE-DUAL STRANGE TALK TOPIC CHAIN IX STABLE OR BREAK

AUTUMN POSS 1 CHILD IX WIND LOVE

AUTUMN POSS ${ }_{1}$ BROTHER IX WIND LOVE

RESTAURANT CHEF IX HOLE STUMBLE-IN $\mathrm{CL}_{\mathrm{CL}}$ RESTAURANT MAN IX HOLE STUMBLE-IN $\mathrm{CL}_{\mathrm{C}}$

CHILD IX POT POSS ${ }_{3 \mathrm{a}}$ HEAD PUT-ON $\mathrm{CL}_{\mathrm{CL}}$ CHILD IX BOWL POSS $3 \mathrm{a}$ HEAD PUT-ON

EGYPT HOTEL IX LOUNGER IX GOAT STAND-ON ${ }_{\mathrm{CL}}$ EGYPT HOTEL IX TABLE IX GOAT STAND-ON ${ }_{\mathrm{CL}}$ 
Prim: Lupe / Hupe Control: Batterie / Hupe

Prim: Macht / Nacht Control: Krieg / Nacht

Prim: Nonne / Sonne

Control: Mönch / Sonne

Prim: Rost / Post

Control: Loch / Post

Prim: Regel / Segel

Control: Erfahrung / Segel

Prim: Riese / Wiese

Control: Mann / Wiese

Prim: Kasse / Tasse

Control: Theke / Tasse

Prim: Turm / Wurm

Control: Schloss / Wurm

Prim: Welt / Zelt

Control: Europa / Zelt

Prim: Wolf / Golf

Control: Hase / Golf
PLAY CAR IX CHILD MAGNIFIER FOR HORN NEED

PLAY CAR IX CHILD BATTERY FOR HORN NEED

MANY KING POSS ${ }_{3}$ POWER OFTEN NIGHT PLAN

MANY KING POSS 3 WAR OFTEN NIGHT PLAN

CLOISTER IX NUN LOVE SUN LOOK-AT

CLOISTER IX MONK LOVE SUN LOOK-AT

LETTERBOX MUCH RUST IX POST BAD FOR LETTERBOX MUCH HOLE IX POST BAD FOR

POSS $_{1}$ GRANDPA MANY RULE FOR SAIL SETTING KNOW POSS $_{1}$ GRANDPA MANY EXPERIENCE FOR SAIL SETTING HAVE

CHILDREN\#TALE GIANT IX GRASSLAND STAMPERS-ACCROSS $\mathrm{CL}_{\mathrm{CL}}$ CHILDREN\#TALE MAN IX GRASSLAND STAMPERS-ACCROSS ${ }_{\mathrm{CL}}$

KITCHEN STORE WOMAN CHECKOUT IX CUP PAY

KITCHEN STORE WOMAN COUNTER IX CUP PAY

FAIRYTALE WOODS TOWER IX WORM CRAWL-UP ${ }_{C L}$ FAIRYTALE WOODS CASTLE IX WORM CRAWL-UP ${ }_{C L}$

LAST YEAR POSS ${ }_{1}$ FRIEND WORLD WITH TENT TRAVEL LAST YEAR POSS ${ }_{1}$ FRIEND EUROPE WITH TENT TRAVEL

ANIMAL STORY CONTENT WOLF IX GOLF PLAY WISH ANIMAL STORY CONTENT RABBIT IX GOLF PLAY WISH 